\title{
Large current driven domain wall mobility and gate tuning of coercivity in ferrimagnetic Mn4N thin films
}

Toshiki Gushi ${ }^{1,2}$, Matic Jovičević Klug ${ }^{3,-}$, José Peña Garcia ${ }^{3}$, Sambit Ghosh², Jean-Philippe Attané2, Hanako Okuno ${ }^{4}$, Olivier Fruchart ${ }^{2}$, Jan Vogel ${ }^{3}$, Takashi Suemasu ${ }^{1}$, Stefania Pizzini ${ }^{*, 3}$, Laurent Vila ${ }^{+, 2}$

1. Institute of Applied Physics, Graduate School of Pure and Applied Sciences, University of Tsukuba, Tsukuba, Ibaraki 305-8573, Japan

2. Univ. Grenoble Alpes, CEA, CNRS, Grenoble INP, INAC, SPINTEC, F-38000 Grenoble, France

3. Univ. Grenoble Alpes, CNRS, Institut Néel, F-38042 Grenoble, France

4. Univ. Grenoble Alpes, CEA, INAC, MEM, F-38000 Grenoble, France

\section{*stefania.pizzini@neel.cnrs.fr}

\section{${ }^{\dagger} \underline{\text { laurent.vila@cea.fr }}$}

־Permanent address: Kiel University, Institute for Materials Science, Kaiserstraße 2, 24143 Kiel, Germany

\section{Abstract}

Spintronics, which is the basis of a low-power, beyond-CMOS technology for computational and memory devices, remains up to now entirely based on critical materials such as $\mathrm{Co}$, heavy metals and rare-earths. Here, we show that $\mathrm{Mn}_{4} \mathrm{~N}$, a rare-earth free ferrimagnet made of abundant elements, is an exciting candidate for the development of sustainable spintronics devices. $\mathrm{Mn}_{4} \mathrm{~N}$ thin films grown epitaxially on $\mathrm{SrTiO}_{3}$ substrates possess remarkable properties, such as a perpendicular magnetisation, a very high extraordinary Hall angle $(2 \%)$ and smooth domain walls, at the millimeter scale. Moreover, domain walls can be moved at record speeds by spin polarised currents, in absence of spin-orbit torques. This can be explained by the large efficiency of the adiabatic spin transfer torque, due to the conjunction of a reduced magnetisation and a large spin polarisation. Finally, we show that the application of gate voltages through the $\mathrm{SrTiO}_{3}$ substrates allows modulating the $\mathrm{Mn}_{4} \mathrm{~N}$ coercive field with a large efficiency.

Keywords: spintronics, spin transfer torque, magnetic domain walls, nitrides thin films, Kerr microscopy, micromagnetism 


\section{Graphic for the Table of Contents}
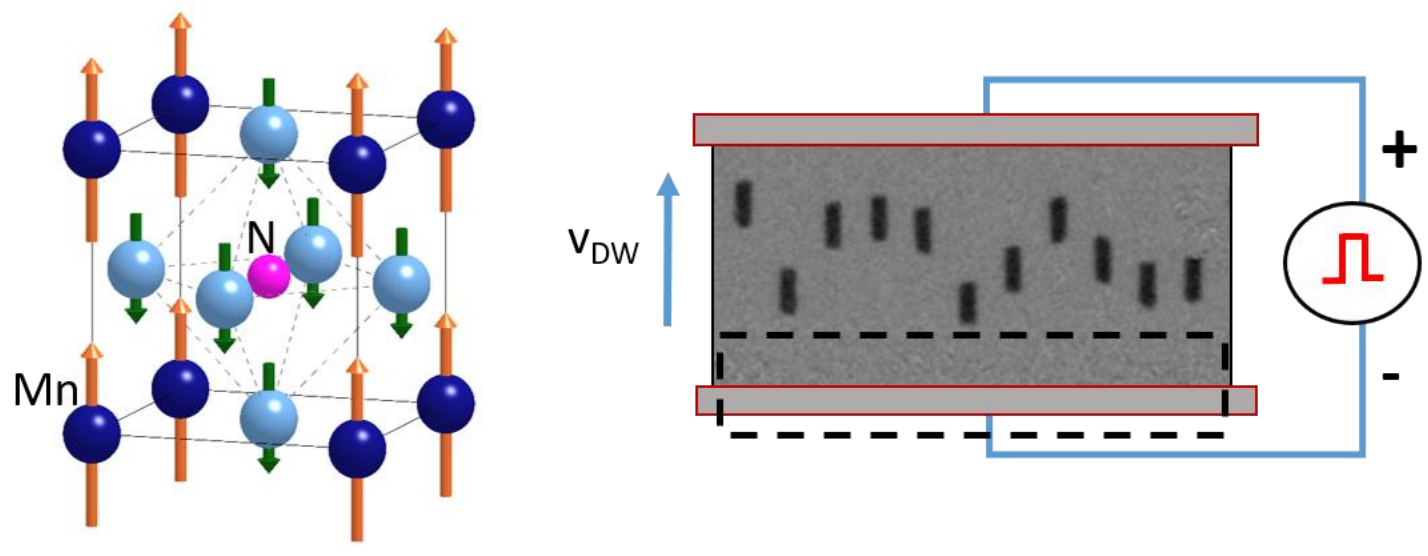

The development of artificial intelligence and big data require the development of high-speed and lowpower memories and processors. In this context, spintronics, which aims at using the electron spin to carry and manipulate data, possesses compelling advantages over competing technologies: intrinsic non-volatility, room-temperature operation, and compatibility with the CMOS technology. It has thus been shown to possess the potential to disrupt not only the memory market, with the on-going commercialization of non-volatile magnetic memories such as MRAMs, but actually the whole information technology market, through the creation of post-CMOS ${ }^{1}$ or of neuromorphic technologies ${ }^{2}$.

Spintronics Achilles' heel might be its hazardous dependence on cobalt, rare-earth elements, and heavy metals (especially tungsten and platinum). These materials are indeed based on elements identified as critical by the government agencies of developed countries, because of the likelihood and impact of supply shortfalls, and of various geopolitical and environmental factors ${ }^{3,4}$.

In this work, we will describe the magnetic properties of a material, made of abundant and cheap elements, and show that it is a promising candidate for the development of a sustainable spintronics: epitaxial $\mathrm{Mn}_{4} \mathrm{~N}$ films. Beyond features such as a perpendicular magnetisation, or a large Extraordinary Hall Effect (EHE), we show that in this material record current driven domain wall velocities can be obtained at room temperature. Whereas in the past years the whole spintronics community shifted its focus from spin-transfer torques to spin-orbit torques, these results show that classical spin-transfer torques remain highly competitive for current-induced DW motion. Also, we show that the coercivity of $\mathrm{Mn}_{4} \mathrm{~N}$ films can be easily tuned by a gate voltage, this ability constituting a tool for DWs, nanomagnets or skyrmion manipulation.

$\mathrm{Mn}_{4} \mathrm{~N}$ is one of the few known magnetic nitrides. While bulk magnetic nitrides have been studied in the 1960's and 1970' $\mathrm{s}^{5}$, only a few groups have succeeded in growing magnetic nitrides thin films, on $\mathrm{MgO}$ or $\mathrm{SrTiO}_{3}$ (STO) substrates, using molecular beam epitaxy ${ }^{6,7}$, pulsed laser deposition ${ }^{8}$ or magnetron sputtering ${ }^{9}$. They grow in the anti-perovskite structure depicted in Figure $1 \mathrm{a}$, with a central $\mathrm{N}$ atom, surrounded by metal atoms located on two inequivalent atomic sites, and antiferromagnetically coupled.

These films present a very large technological interest: they are made of only abundant elements, which responds to the increasing need for sustainable and low cost electronics. They are ferrimagnets 
with a low spontaneous magnetisation $M_{s}$, which should allow increasing the efficiency of spin transfer and spin orbit torques. This should facilitate magnetisation manipulation, increase the switching speed and lower the power consumption of spintronic devices. The small spontaneous magnetisation also provides a relatively small sensitivity to externally applied magnetic fields: ferrimagnetic-based devices do not need to be magnetically shielded when embedded in memory or computational devices, and the production of stray fields is minimized. The Curie temperature of the bulk compound is $745 \mathrm{~K}^{10}$.

Among the magnetic nitride series, $\mathrm{Mn}_{4} \mathrm{~N}$ holds a characteristic that is much looked for in spintronics: in appropriate growth conditions, it presents a perpendicular magnetisation. This property, associated to the low $\mathrm{M}_{\mathrm{s}}$, allows foreseeing high current-driven domain wall speeds by spin transfer torques ${ }^{11}$.

Here, $10 \mathrm{~nm}$ thick films were grown epitaxially on an STO(001) substrate. As recently observed ${ }^{12}$, the small lattice mismatch between the film and the substrate allows obtaining a high crystalline quality. The X-Ray Diffraction (XRD) spectra of Figure $1 \mathrm{~b}$ demonstrates the epitaxial growth on the STO substrate with a (001) texture. The quality of this epitaxial growth is also evidenced by $\omega$-scan rocking curves and RHEED diffraction (cf. Methods). The typical RMS roughness of the layer, measured by Atomic Force Microscopy, is smaller than $0.7 \mathrm{~nm}$. The Scanning Transmission Electron Microscopy images of the $\mathrm{Mn}_{4} \mathrm{~N}$ epilayer, shown in Figures $1 \mathrm{c}$ and $1 \mathrm{~d}$, give further evidence of its high crystalline quality, the absence of dislocations and the perfect epitaxy and its sharp interface with the Ti terminated STO.

The magnetic properties of the films were studied by Vibrating Sample Magnetometry, SQUID and transport measurements. The extracted spontaneous magnetisation is small $\left(M_{s}=7.1 \times 10^{4} \mathrm{~A} / \mathrm{m}\right)$, whereas the uniaxial magnetic anisotropy is large enough $\left(\mathrm{K}_{\mathrm{u}}=1.1 \times 10^{5} \mathrm{~J} / \mathrm{m}^{3}\right)$ to obtain perpendicular magnetisation. The hysteresis loop measured by extraordinary Hall effect (Figure 1e) shows 100\% remanence. As the magnetic field acts on the magnetisation through a $-\mu_{O} \vec{H} . \vec{M}$ Zeeman energy density, one can expect this material, which possesses a small $\mathrm{M}_{\mathrm{s}}$, to exhibit a large coercive field. The coercivity remains however quite low $(170 \mathrm{mT})$.

Also, Magneto-Optical Kerr microscopy observations of the magnetic domain structure have been made for partially reversed states (cf. Figure 1.f). The observed scarce nucleation centers with remarkably large domains, at the millimeter scale, with smooth and very long DWs ${ }^{12}$, result from the high crystalline quality. 

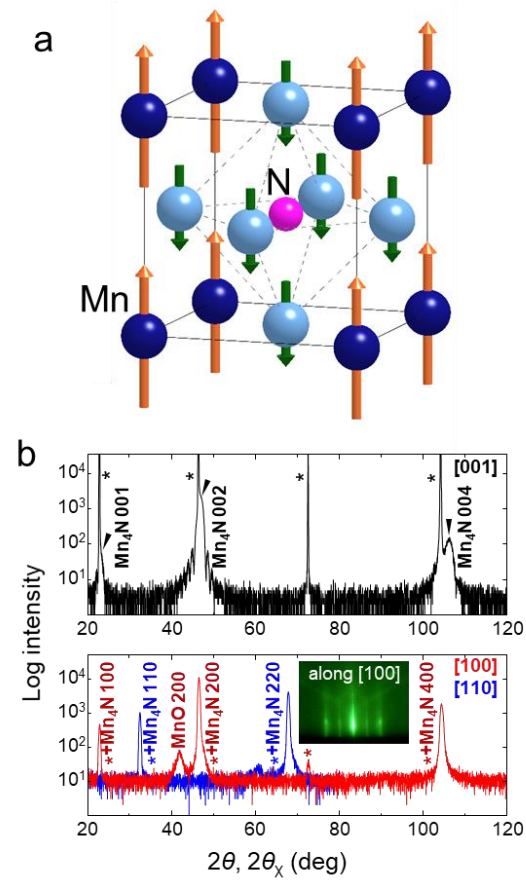

C

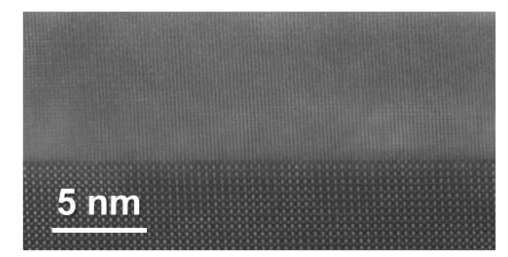

d

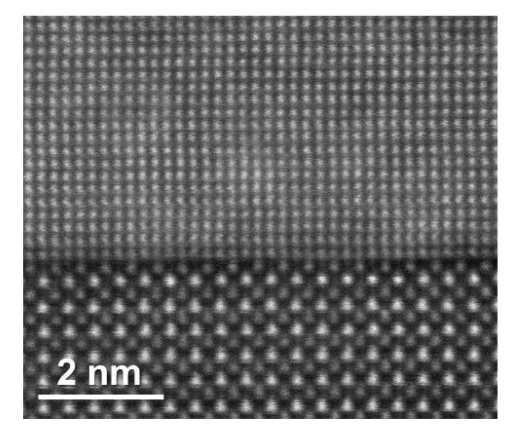

e

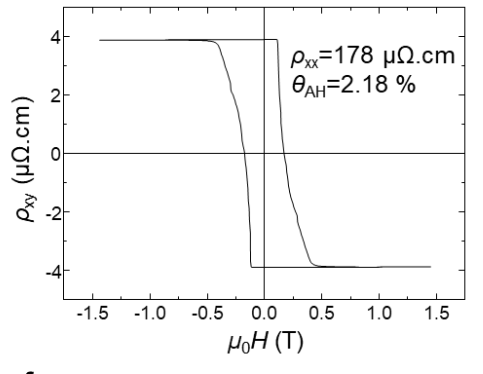

$f$

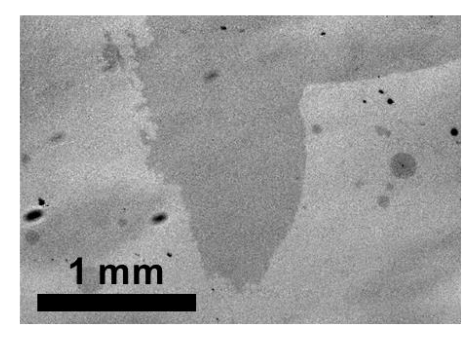

Figure 1 (a) Scheme of the anti-perovskite structure of $\mathrm{Mn}_{4} \mathrm{~N}$ films. The metallic ions located the corners and at the centers of the faces are antiferromagnetically coupled. (b) Out-of-plane (top) and in-plane (bottom) XRD spectra of a $\mathrm{Mn}_{4} \mathrm{~N}(10 \mathrm{~nm}) / \mathrm{STO}$ deposit, with Kiessig fringes testifying of the crystalline quality. The in-plane XRD measurements were performed by setting the scattering vector along STO[100] (red) and STO[110] (blue). Asterisks correspond to the STO substrate diffraction peaks. (c) Scanning Transmission Electronic image of the $\mathrm{Mn}_{4} \mathrm{~N} / \mathrm{STO}$ sample corroborating the crystalline quality of $\mathrm{Mn}_{4} \mathrm{~N}$. (d) Higher magnification image showing the perfect epitaxy of the $\mathrm{Mn}_{4} \mathrm{~N}$ with the Ti terminated STO interface and its sharpness, any dislocation is observed on the TEM specimen. (e) Hysteresis loop measured by Extraordinary Hall effect, for an applied field perpendicular to the surface. (f) Magneto-optical Kerr microscopy observation of the sample in a partially reversed state of magnetisation ( $\left.M / M_{s}=-0.1\right)$. The reversed domain appears in black.

$\mathrm{Mn}_{4} \mathrm{~N}$ films are metallic, with layer resistivity around $180 \mu \Omega \mathrm{cm}$ at room temperature, comparable to CoFeB-based materials. Although $\mathrm{Mn}_{4} \mathrm{~N}$ is made of only light elements, its Extraordinary Hall Effect angle is large (around $2 \%$ ), comparable to that of materials with strong spin-orbit coupling such as FePt or $\mathrm{TbCoFe}^{13}$. 

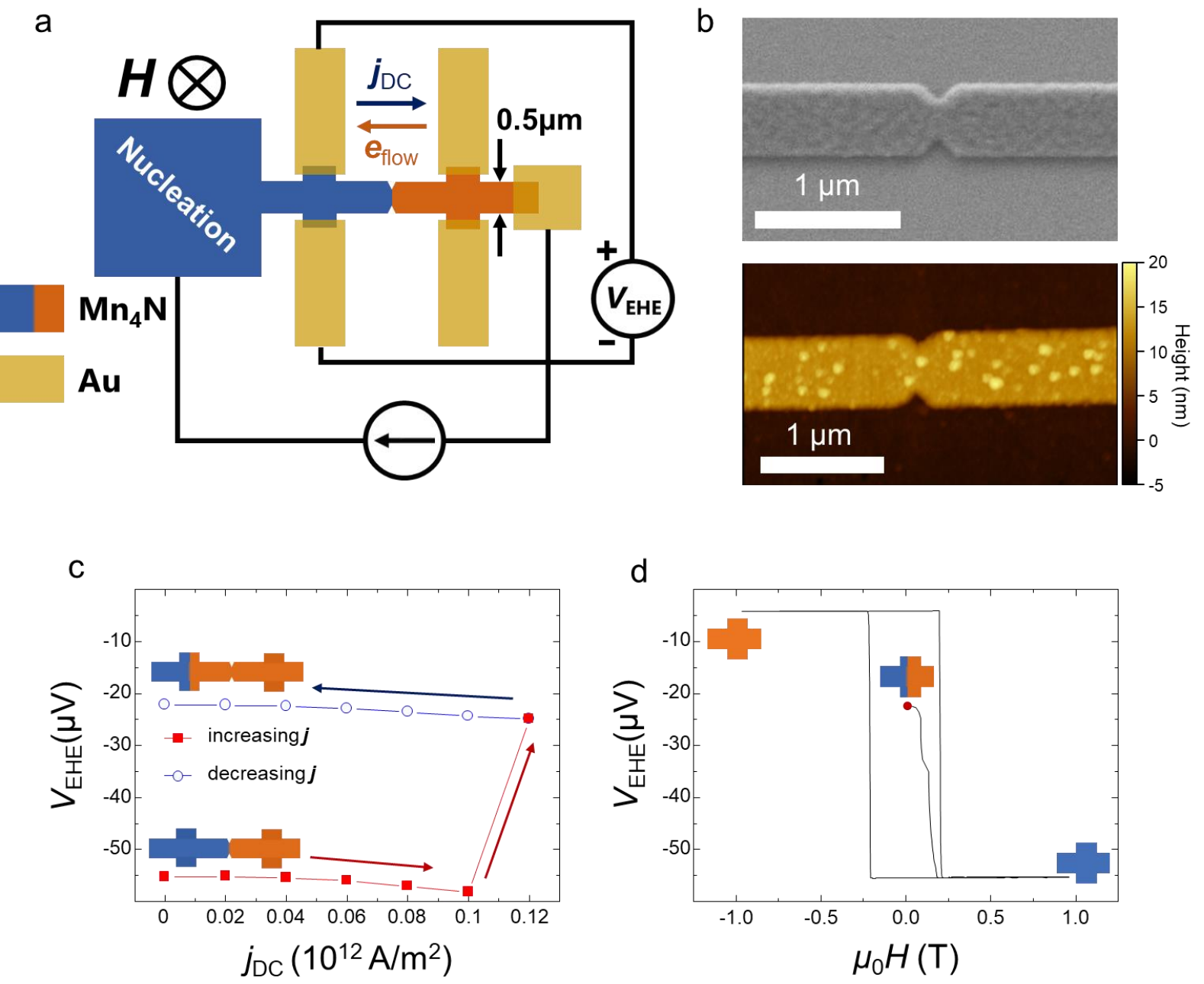

Figure 2 (a) Schematics of the device and of the probe connection (b) SEM images (top) of the constriction before etching, and AFM image (bottom) after etching. (c) Room temperature Hall voltage recorded at the left Hall cross as a function of the applied current density. The DW is firstly pinned on the constriction. It is then depinned by the current, and finally propagates within the left Hall cross, causing a partial reversal (64\%) of the magnetisation. (d) Hall voltage of the first Hall cross obtained when increasing the field from 0 to positive values, the starting point being the final state of fig. (c). This confirms that after the application of the DC current in (c), the DW is indeed located in the Hall cross.

The efficiency of the spin-transfer torque was first studied by extraordinary Hall measurements, using DC currents and quasi-static fields in the device geometry depicted in Figure 2a. As seen in Figure $2 b$, the $\mathrm{Mn}_{4} \mathrm{~N}$ layer was patterned by ion milling into $0.5 \mu \mathrm{m}$ wide Hall bars, with a constriction patterned in between the two Hall crosses (cf. § Methods). The patterning induces a sharpening of the switching, with very square hysteresis loops (cf. Figure $2 \mathrm{~d}$ ). The minor loops show that the threshold field for DW propagation remains low, around $0.15 \mathrm{~T}$. This field can be compared to the critical current density required to induce DW motion. As seen in Figure $2 c$, the application of a DC current allows inducing the DW motion at zero field, for a current density of $1.2 \times 10^{11} \mathrm{~A} / \mathrm{m}^{2}$. The $\mathrm{DW}$ is then depinned from a notch, and propagates over more than $5 \mu \mathrm{m}$ before getting pinned at the cross. The spin transfer efficiency, i.e., the ratio between the typical propagation field and the $D C$ current required to induce DW motion, is thus around $1.25 \times 10^{-12} \mathrm{Tm}^{2} / \mathrm{A}$. This value is two orders of magnitude higher than that of NiFe, and similar to that of systems with heavy elements and large spin-orbit coupling such as FePt ${ }^{14}$, or of TbCoFe and CoGd ferrimagnets ${ }^{15}$. 
Note that in most magnetic thin films studied in the last years, the DWs were moved by the Spin-Orbit Torque (SOT) arising from the growth on a Spin-Hall effect material, and from the presence of the interfacial Dzyaloshinskii-Moriya interaction (DMI) which favours chiral Néel walls ${ }^{16,17,18}$. On the contrary, DW motion is driven here by the classical Spin Transfer Torque (STT): since the $\mathrm{Mn}_{4} \mathrm{~N}$ layer is thick $(10 \mathrm{~nm})$, interfacial effects should be negligible, even in the presence of an interfacial DMI. The DW propagates in the direction of the electron flow, which is consistent with this interpretation. In order to rule out the contribution of spin-orbit torques to the DW dynamics, we carried out Kerr microscopy measurements of domain wall motion under in-plane magnetic field, which demonstrated the absence of DMI in our system (cf. Supporting Information).

The efficiency of the spin transfer torque was also investigated by measuring directly current-driven DW velocities by Kerr microscopy as shown in Figure 3a and $3 \mathrm{~b}$. A $10 \mathrm{~nm}$ thick $\mathrm{Mn}_{4} \mathrm{~N}$ layer was patterned into $10 \mu \mathrm{m}$ long, $2 \mu \mathrm{m}$ and $1 \mu \mathrm{m}$ wide strips. Several wires were connected in parallel via a large injection pad, in order to optimize the impedance matching with the voltage generator, and to obtain a statistically relevant measurement of the DW velocity.

DWs can be created using an out-of-plane field pulse, leading to one DW per wire. Current pulses of durations ranging from 1 to $3 \mathrm{~ns}$, with current densities up to $1.5 \times 10^{12} \mathrm{~A} / \mathrm{m}^{2}$ were then applied to induce DW motion. The differential images of Figures $3 \mathrm{a}$ and $3 \mathrm{~b}$ allow measuring the distance over which the DWs have propagated during the pulse, therefore giving access to the average DW velocity, for $2 \mu \mathrm{m}$ and $1 \mu \mathrm{m}$ wide strips respectively. The limited dispersion of the DW displacements among the nanowires points out that DWs are weakly pinned by defects (i.e., the behaviour is not stochastic).

Figure $3 c$ shows the variation of the domain wall velocity as a function of the current density. The behaviours are nearly identical for $1 \mu \mathrm{m}$ and $2 \mu \mathrm{m}$ wide strips. For low current densities $\mathrm{J}$, in the thermally activated regime where the DW movement is hindered by defects, the speed changes exponentially with J. Above around $J=8.5 \times 10^{11} \mathrm{~A} / \mathrm{m}^{2}$, the DW speed starts varying linearly with $\mathrm{J}$, and very high DW velocities (up to $900 \mathrm{~m} / \mathrm{s}$ ) are reached for $1.3 \times 10^{12} \mathrm{~A} / \mathrm{m}^{2}$, in the direction of the electron flow.

Such high DW velocities deserve some discussion. Current driven domain wall motion by STT has been observed in thin films with both in-plane ${ }^{19,20}$ and out-of-plane magnetisation. In in-plane magnetized films of permalloy, the measured DW velocities are typically of the order of $100 \mathrm{~m} / \mathrm{s}$, for currents densities around $1.5 \times 10^{12} \mathrm{~A} / \mathrm{m}^{2}$. However, in these systems the DW motion is largely affected by pinning, and in general a wide dispersion of DW displacements is observed even in the flow regime ${ }^{20}$. 


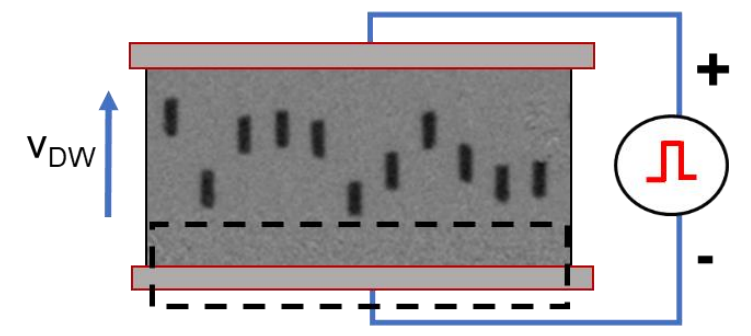

C

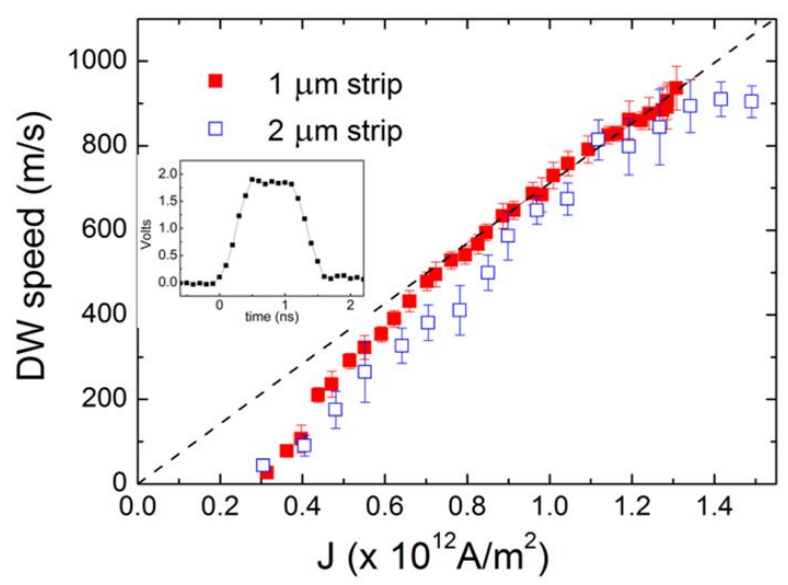

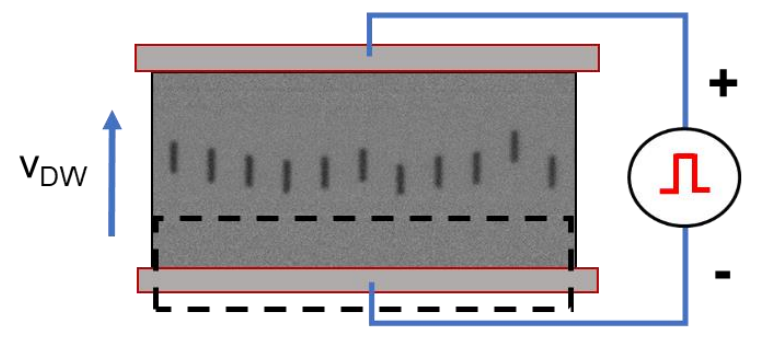

d

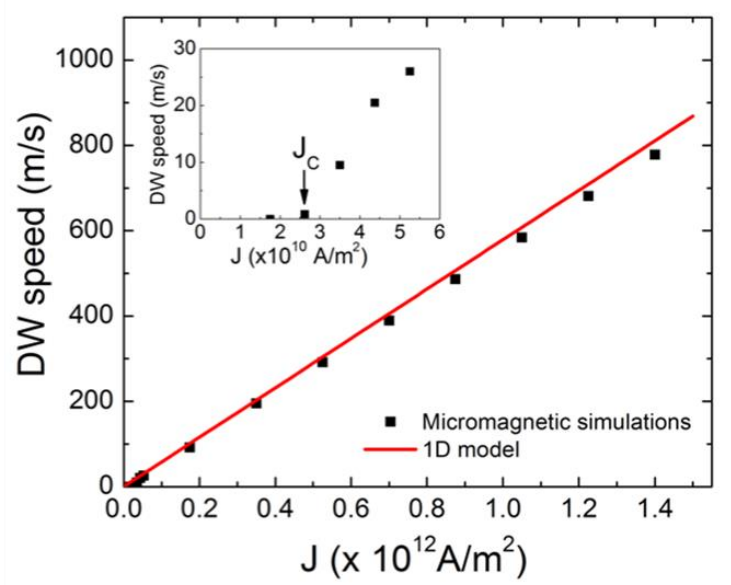

Figure 3 (a) and (b): differential MOKE images showing the displacement of several DWs in 2 um (a) and 1um wide $\mathrm{Mn}_{4} \mathrm{~N}$ strips (b) after $10 \times 1 \mathrm{~ns}$ current pulses. The current density is respectively $\sim 0.4$ and $\sim 1.05 \times 10^{12} \mathrm{~A} / \mathrm{m}^{2}$ and the strips are $10 \mu \mathrm{m}$ long. The black contrast corresponds to the zone over which the DWs moved during the current pulses. (c) Domain wall velocity vs. current density measured for 1 and $2 \mu \mathrm{m}$-wide strips. The domain wall velocity reaches an average above $900 \mathrm{~m} / \mathrm{s}$ at $1.3 \times 10^{12} \mathrm{~A} / \mathrm{m}^{2}$. The dotted line is a guide to the eye to emphasize the linear regime. The inset shows an example of $1 \mathrm{~ns}$ current pulse measured with an oscilloscope in series with the sample. (d) Theoretical DW velocity (red line) obtained using the 1D model (equation 3) using the experimental magnetic parameters $\left(\mathrm{M}_{\mathrm{s}}\right.$ and $\mathrm{K}_{\mathrm{u}}$ ) and taking $\mathrm{P}=0.8$ (giving the best agreement between experimental and theoretical DW mobility, see below) and $\alpha=0.15$ as determined by time resolved Kerr measurements. The black dots are the results of micromagnetic simulations using the same parameters. The inset is a zoom of the low current density regime.

Moreover, since DWs are wide $(>100 \mathrm{~nm})$ in these in-plane systems, the interest for applications to logic and storage devices is limited. Quite high spin transfer efficiencies have been measured in systems with perpendicular magnetic anisotropy (PMA) such as $\mathrm{Co} / \mathrm{Ni}$ or $\mathrm{FePt}^{21}$, but in these systems DW motion at zero field is usually hindered by pinning, which induces a large coercivity and a stochastic behaviour. In Pt/Co/Pt, DWs could not be moved by STT, due to the limited spin polarisation within the Co layer ${ }^{22}$. On the other hand, very high DW mobilities were found in magnetic semiconductor films such as $(\mathrm{Ga}, \mathrm{Mn})(\mathrm{As}, \mathrm{P})$ for very low current densities ${ }^{23,24}$. However these measurements were done at low temperature, and the current density was limited to some $10^{10} \mathrm{~A} / \mathrm{m}^{2}$, with a maximum speed of $30 \mathrm{~m} / \mathrm{s}$ at $10^{10} \mathrm{~A} / \mathrm{m}^{2}$.

Although in recent years the community has focused nearly entirely on SOT-driven DW dynamics, the giant DW velocities obtained in $\mathrm{Mn}_{4} \mathrm{~N}$, show that the STT can be competitive with SOT. SOT has been 
found to be an efficient mechanism to drive DWs in non-centrosymmetric multilayers, in which a FM is deposited on a heavy metal like $\mathrm{Pt}^{17,18}$. The prototypical example is $\mathrm{Pt} / \mathrm{Co}(0.6 \mathrm{~nm}) / \mathrm{AlOx}^{25}$ where the chiral Néel structure acquired by the DWs in the presence of interfacial Dzyaloshinskii-Moriya interaction ${ }^{16}$ leads to a high mobility $\left(v=400 \mathrm{~m} / \mathrm{s}\right.$ for $\left.\mathrm{J}=3 \times 10^{12} \mathrm{~A} / \mathrm{m}^{2}\right)$ with a satisfying reproducibility of the DW movements. Higher SOT-driven DW mobilities were obtained recently in a multilayer structure in which two $\mathrm{Co} / \mathrm{Ni}$ layers were coupled anti-ferromagnetically through a Ru layer ${ }^{26}$, or in GdCo ferrimagnet layers deposited on top of $\mathrm{Pt}^{27}$. In these systems the $\mathrm{DW}$ velocity was clearly observed to vary with the total magnetisation. In the former experiment, a maximum velocity of $v=750 \mathrm{~m} / \mathrm{s}$ for $\mathrm{J}=3 \times 10^{12} \mathrm{~A} / \mathrm{m}^{2}$ was obtained at room temperature using a $\mathrm{Ru}$ spacer layer, so that the stack magnetisation was reduced to $8 \%$ of the spontaneous magnetisation of a single Co/Ni layer. In the latter one, a peak velocity of $1250 \mathrm{~m} / \mathrm{s}$ was reported at $240 \mathrm{~K}$ (the angular moment compensation temperature) for a current density of $2 \times 10^{12} \mathrm{~A} / \mathrm{m}^{2}$.

In this context, the velocities presented here at room temperature for DWs in $\mathrm{Mn}_{4} \mathrm{~N}$ are comparable to the best results obtained using SOTs. Moreover, they represent the first demonstration of very efficient current-driven DW motion by pure STT using a system with PMA.

In order to understand the microscopic origins of the high current-induced DW velocities achieved in $\mathrm{Mn}_{4} \mathrm{~N}$ thin films, we have considered the analytical expression of the STT in the adiabatic limit. In a perfect nanowire with out-of-plane magnetisation, the $1 \mathrm{D}$ model describes well the main features of the DW velocity under a spin-polarised current ${ }^{28,29}$.

Magnetisation dynamics is governed by the Landau-Lifshitz-Gilbert equation (LLG), with additional terms taking into account the effect of spin-polarised current on the magnetisation. In ferrimagnetic systems, the LLG equation needs to be modified close to the compensation temperature when the Landé factors of the two magnetic sublattices are very different ${ }^{27,30}$. Since the orbital moment of $\mathrm{Mn}$ is close to zero, the Landé factors should be very similar (close to 2) for both sublattices in $\mathrm{Mn}_{4} \mathrm{~N}$. Moreover, the temperature variation of the magnetization does not reveal any compensation temperature. The DW dynamics close to room temperature should thus be very similar to the dynamics of a ferromagnetic system ${ }^{31}$. In our system with PMA and negligible Dzyaloshinskii-Moriya interaction, the DWs are expected to be in the Bloch configuration at rest (see Supporting Information). In the adiabatic limit of current-driven dynamics, the DW can move continuously only when its magnetisation can start precessing in order to align with the spin polarisation of the incoming conduction electrons (i.e., along $z$ in a system with PMA). To do so, the DW energy has to overcome the anisotropy energy $K_{D}$ given by the difference in energy between the Bloch and the Néel DW configurations. This occurs above a threshold critical current density $J_{c}$ :

$$
J_{c}=\frac{2 e}{\hbar P} \Delta(\varphi) K_{D}
$$

where $\mathrm{P}$ is the spin polarisation of the conduction electrons, $\Delta(\varphi)$ is the DW width as a function of the tilt angle $\varphi$, the angle between the magnetisation in the center of the DW and the DW plane. The anisotropy energy $K_{D}$ depends on the sample geometry and on the spontaneous magnetisation (see Supporting Information). Using the experimental strip geometry ( $1 \mu \mathrm{m}$ wide and $10 \mathrm{~nm}$ thick) and the material parameters measured experimentally for the $\mathrm{Mn}_{4} \mathrm{~N}$ film $\left(M_{S}=7.1 \times 10^{4} \mathrm{~A} / \mathrm{m}, K_{u}=\right.$ $0.11 \times 10^{6} \mathrm{~J} / \mathrm{m}^{3}$ and taking $A=10 \mathrm{pJ} / \mathrm{m}$ (value extracted by scaling the Curie temperature to that of 
other nitride compounds) and $\mathrm{P}=0.8$, the critical current density is expected to be $J_{c} \approx 1.9 \times 10^{10}$ $\mathrm{A} / \mathrm{m}^{2}$. This is much lower than that of permalloy strips with in-plane magnetisation $\left(J_{c} \approx 10^{13} \mathrm{~A} / \mathrm{m}^{2}\right)^{11}$. While a non-adiabatic torque has been invoked to explain the DW motion observed experimentally in permalloy strips well below $J_{c}$, in our case the observed DW motion occurs for current densities well above Jc and can therefore be explained in terms of the adiabatic torque alone. ${ }^{32,33}$.

As seen in the Supporting Information, when $J \gg J_{C}$ the adiabatic torque drives the DWs with a velocity given by:

$$
\boldsymbol{v} \approx \frac{1}{1+\alpha^{2}}|\boldsymbol{u}|
$$

where $\boldsymbol{u}=\frac{g \mu_{B}}{2 e M_{S}} P \boldsymbol{J}$ is the spin-drift velocity, parallel to the electron flow.

From Equation 2, the mobility $v / J$ is proportional to the ratio $\mathrm{P} / \mathrm{M}_{\mathrm{s}}$ of the spin polarisation and the spontaneous magnetisation, with a negligible dependence on the damping parameter, $\alpha=0.15$ as measured by time-resolved Kerr spectroscopy. The spin polarisation $P$ is then the only fitting parameter and an estimation of its value in $\mathrm{Mn}_{4} \mathrm{~N}$ can be obtained from the comparison between the experimental DW velocities and the result of Equation 2 in which $P$ is varied. Experimentally, a linear regime indeed appears in the speed versus current density curve above the thermally activated regime. The best agreement between experiments (Figure 3c) and 1D model (Figure 3d) is obtained using a polarisation value around $\mathrm{P}=0.8(+/-0.1)$, close to the polarisation of the density of states obtained using first-principle calculations ${ }^{34}$. We can then conclude that the large domain wall speeds observed for $\mathrm{Mn}_{4} \mathrm{~N}$ are due to the conjunction of the small value of the spontaneous magnetisation and of the high spin polarisation.

In order to confirm the different features predicted by the 1D model we have also performed micromagnetic simulations (see Supporting Information). The results reported in Figure $3 \mathrm{~d}$ show an overall agreement of the $1 \mathrm{D}$ model with the $2 \mathrm{D}$ simulations.

Note that in our simulations we have neglected the effect of the non-adiabatic torque on the DW velocity. While this term had to be introduced to explain the DW dynamics in in-plane magnetized systems ${ }^{19}$ below the intrinsic depinning current density, it has actually a much lower influence on the DW velocity in our system.

Below the critical current density $J_{c}$, the presence of the non-adiabatic torque results in a steady regime motion, with a velocity $v=\frac{\beta}{\alpha} u$. This effect is not observable in our system, as the critical current $J_{c}$ is extremely small and obscured by the creep regime. Above $J_{c}$, in the precessional regime, a term $\frac{a \beta}{1+a^{2}} u$ has to be added to the velocity of Equation 2. However, since $\alpha$ is much smaller than unity, this extra term is negligible with respect to the adiabatic term. Although the measurement of the non-adiabatic term has been the subject of severe debates ${ }^{15}$, its main effect is to explain the presence of DW motion below the critical current density. Here we show that the adiabatic torque alone can explain the measured DW speeds down to low current densities, because of the small critical current densities characteristic of PMA systems with small Ms. This point is further discussed in the Supporting Information, where we also study the hypothesis according to which $\alpha=\beta$. 
The last property we would like to address is the effect of the electric field on the anisotropy of the $\mathrm{Mn}_{4} \mathrm{~N}$ layer shown in Figure 4. A back gate voltage was applied to the bottom of the $300 \mu \mathrm{m}$ STO substrate, while measuring the coercivity of a $4.2 \mathrm{~nm}$-thick $\mathrm{Mn}_{4} \mathrm{~N}$ layer with a magnetic field (cf. $\S$ Methods). The measurements were performed at $10 \mathrm{~K}$, so that the dielectric constant of STO reaches very high values $(30000)$. The gate voltage acts on both the negative and positive parts of the magnetic hysteresis, the coercivity varying linearly with the applied voltage, see Figure $4 \mathrm{e}$. By assuming that the coercivity variations correspond to variations of the anisotropy energy $y^{35}$, the efficiency of the electric field effect can be written $\eta=\frac{d E_{k} / S}{d E_{\text {Gate }}}=\frac{E_{k} / S}{H_{C}} \frac{d H_{C}}{d E_{\text {Gate }}}$, where $E_{k} / S$ is the anisotropy energy per surface, and $H_{C}$ the coercive field. In our system, $\eta$ reaches a value of $-15 \mathrm{pJ} /(\mathrm{V} . \mathrm{m})$. This very large efficiency, which could be further enhanced by using thinner $\mathrm{Mn}_{4} \mathrm{~N}$ films, requires further in-depth studies to be understood. In particular, it is possible that a strain-based mechanism adds up to the charge transfer mechanism, due to the STO substrate behaviour in the low-temperature tetragonal phase $36,37,38$. Whatever the mechanism, this value is up to now only approached in systems with much thinner magnetic layers ${ }^{39}$.

a
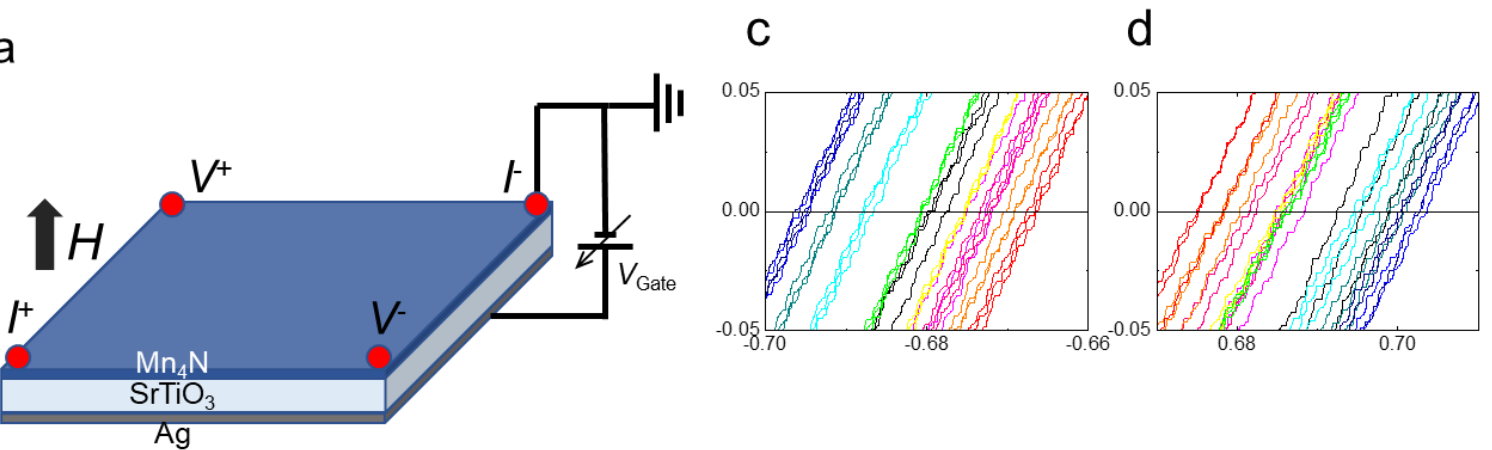

b

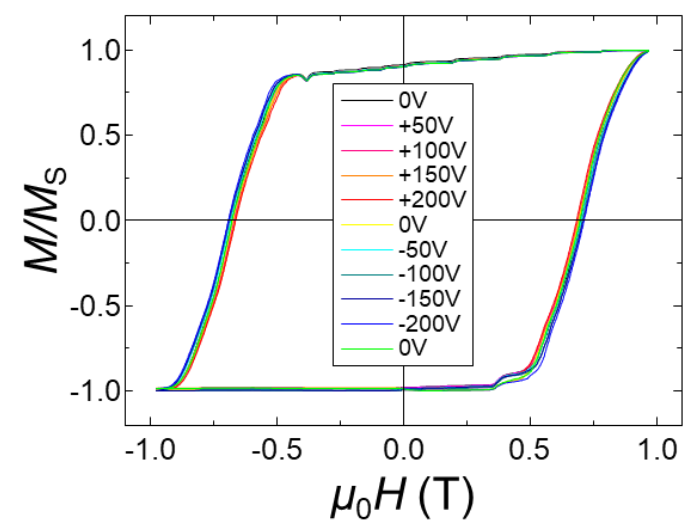

e

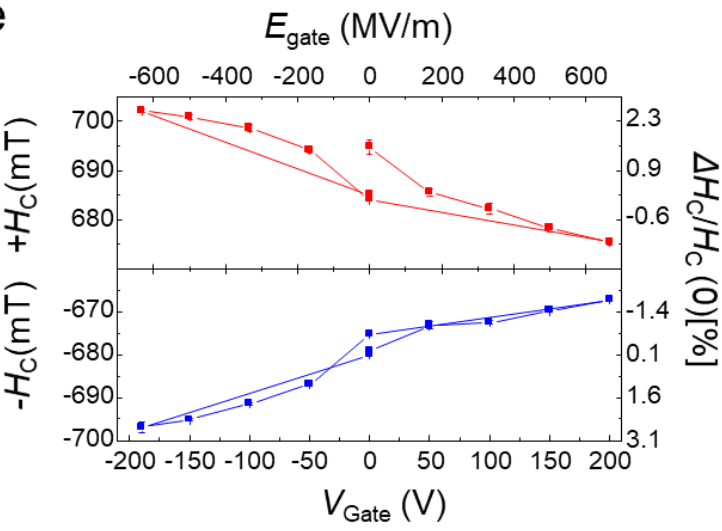

Figure 4 (a) Schematics of the experiments. A gate voltage is applied to the back of the STO substrate, while the top electrical contacts, arranged in a Van der Pauw configuration, are used to measure the EHE of the $\mathrm{Mn}_{4} \mathrm{~N}$ layer. (b) Hysteresis loops recorded for various applied back gate voltages. (c) and (d) Zoom of the figure b close to the coercive field, for negative (c) and positive (d) applied fields, using the same color code for the gate voltage. (d) Evolution of the coercivity with the applied gate voltage, for both the positive (top) and negative (bottom) halfloop.

\section{Conclusion}


In conclusion, $\mathrm{Mn}_{4} \mathrm{~N} / \mathrm{STO}$ films present several properties that make them compelling candidates for spintronics applications. They are characterized by a perpendicular magnetisation, and notwithstanding the absence of heavy elements with high spin-orbit coupling, they exhibit a large extraordinary Hall effect. We showed in this system efficient current-induced domain wall motion, simply due to the adiabatic spin-transfer torque. The observed domain wall velocities largely surpass those obtained using spin transfer torques up to now, and is at least comparable to the best results reported for non-centrosymmetric stacks with interfacial DMI. We show that the low critical current is a consequence of the PMA and of the low magnetisation of the ferromagnetic material, which leads to a low barrier for precession of the DW magnetisation. The high DW mobility observed in the linear regime is a consequence of the small magnetisation and of the high spin polarisation. Finally, we demonstrate the possibility to control the magnetic anisotropy of $\mathrm{Mn}_{4} \mathrm{~N}$ films using electric fields. As $\mathrm{Mn}_{4} \mathrm{~N}$ is made of cheap and abundant elements, and does not include critical materials such as precious metals and rare-earths, it appears as a worthy candidate for sustainable spintronics applications. Future developments of this work may include the substitution of $\mathrm{Mn}$ by other $3 \mathrm{~d}$ magnetic elements, in order to tailor the magnetic parameters, and for instance to reach the compensation point.

\section{Methods}

Sample growth

The $\mathrm{Mn}_{4} \mathrm{~N}$ thin films were grown on STO(100) substrates by plasma-assisted molecular beam epitaxy (MBE), as in refs..$^{6,40}$. During the deposition, the substrate temperature was kept at $450{ }^{\circ} \mathrm{C}$, to allow the diffusion of $\mathrm{Mn}$ and $\mathrm{N}$ atoms coming from the $\mathrm{Mn}$ solid source of a Knudsen-cell and from the radio-frequency (RF) $\mathrm{N}_{2}$ plasma. We optimised the growth condition by using a $\mathrm{Mn}$ deposition rate of $1.5 \mathrm{~nm} / \mathrm{min}$, a $\mathrm{N}_{2}$ gas flow of $1.0 \mathrm{~cm}^{3} / \mathrm{min}$, a pressure in the chamber of $4.5 \times 10^{-3} \mathrm{~Pa}$, and an input power of $105 \mathrm{~W}$. The samples were capped with $\mathrm{SiO}_{2}$ for the CIDWM experiments, and with Ta for the voltagecontrol of the magnetic anisotropy experiments, using a sputtering gun installed in the MBE chamber.

\section{XRD/RHEED}

The $\mathrm{Mn}_{4} \mathrm{~N}$ films were characterized in-situ by RHEED, with the $20 \mathrm{keV}$ electron beam azimuth along the STO [100] direction. The diffraction pattern of anti-perovskite nitride consists of fundamental and superlattice diffraction peaks, similarly to what has been simulated for $\mathrm{Fe}_{4} \mathrm{~N}$ thin film ${ }^{41}$. Our sample showed streaky RHEED patterns with obvious superlattice diffraction lines, which are sharper and surrounding the central line, indicating the presence of well ordered $\mathrm{N}$ atoms at the body center of the fcc Mn lattice.

The crystalline quality was also evaluated by $\omega-2 \vartheta$ (out-of-plane), $\varphi-2 \vartheta_{\chi}$ (in-plane) XRD and $\omega$-rocking curve measurement, using $\mathrm{Cu}$ K $\alpha$ radiation in a Rigaku Smart Lab ${ }^{\circledast}$. For the in-plane diffraction, the incidence angle $\omega$ was fixed as $0.4^{\circ}$, and the scattering vector $\boldsymbol{Q}$ was also set along STO [100] and [110]. The $\omega$-rocking curve at the $\mathrm{Mn}_{4} \mathrm{~N}[004]$ peak exhibits a remarkably small full width at half maximum of $0.14^{\circ}$, highlighting the very high crystalline quality of the epilayer ${ }^{12}$. The thickness of the layer was also measured by $\mathrm{X}$-ray reflectometry. 
The surface state was observed using a Bruker Dimension in the oscillating mode. The root mean square roughness $(0.7 \mathrm{~nm})$ has been evaluated on a $4 \times 4 \mu \mathrm{m}^{2}$ area.

TEM

Scanning transmission electron microscopy (STEM) measurements have been carried out using a Cscorrected FEl Themis at $200 \mathrm{keV}$. High angle annular dark field (HAADF)-STEM images were acquired using a convergence semiangle of $20 \mathrm{mrad}$ and collecting scattering $>65 \mathrm{mrad}$. The STEM specimen was prepared by the FIB lift-out technique using a FEl dual-beam Strata $400 \mathrm{~S}$ at $30 \mathrm{kV}$.

VSM/SQUID

The magnetisation and magnetic anisotropy were measured by Quantum design MPMS ${ }^{\circledR}$ with outof-plane (1.5 T) and in-plane ( $7 \mathrm{~T})$ magnetic fields. The anisotropic energy $K_{\mathrm{u}}$ was calculated from the area enclosed in-between the out-of-plane and in-plane magnetisation curves.

EHE and voltage control of the anisotropy

Extraordinary Hall effect loops and resistivity measurements were performed using the Van der Pauw method for blanket layers at $10 \mathrm{~K}$, in a cryostat placed in-between the poles of a $1.2 \mathrm{~T}$ electromagnets. The 4 corners of the sample were wire-bonded using Al wires. A back gate voltage up to $\pm 200 \mathrm{~V}$ was applied by sticking the sample to the sample holder with silver paint.

Domain wall speed measurements

The $\mathrm{Mn}_{4} \mathrm{~N}$ films were processed into 1 and $2-\mu \mathrm{m}$-wide strips by electron beam lithography and $\mathrm{Ar}$ ion milling technique, using lifted-off Al patterns as etching masks. The contact electrodes consist in a $\mathrm{Ti} / \mathrm{Au} / \mathrm{Ti}$ stack. The domain wall velocities were measured using Magneto-Optical Kerr microscopy. After saturation of the magnetisation, high field pulses were applied in the reversed direction to nucleate a domain in the injection pad and to inject domain walls into the nanostrips. The domain walls were then displaced using current pulses generated by a voltage generator, able to provide voltage pulses up to $80 \mathrm{~V}$ in amplitude and down to $500 \mathrm{ps}$ in duration. The domain wall velocities were calculated by dividing the domain wall displacement by the pulse length full width half maxima, measured by an oscilloscope connected in series with the sample. Further verification of the DW speeds was done for some current density values by measuring the DW displacement $\Delta \mathrm{x}$ for different current pulse durations $\Delta t$, and extracting the DW velocity from the slope of the curve $\Delta x v s . \Delta t$.

\section{Acknowledgments}

We acknowledge N. Mollard for the TEM specimen preparation. The devices were prepared in PTA platform from Grenoble, with partial support from the French RENATECH network. This work received support from the French ANR programme through projects OISO (ANR-17-CE24-0026-03), TOPRISE (ANR-16-CE24-0017) and the Laboratoire d'Excellence LANEF (ANR-10-LABX-51-01) in Grenoble. TG acknowledge JSAP support. JPB acknowledges the European Union's Horizon 2020 research and innovation programme under the Marie Skłodowska-Curie grant agreement No 754303. We acknowledge Prof. Isogami from the NIMS in Japan, for the measurement of the damping parameter. 


\section{Author contributions}

The experiments were performed by T. G., M. J. K. and S.G., and the simulations by J. P. G. H. O. performed the TEM study. The work has been supervised by L.V., T.S., O.F., S.P., J.P.A. and J.V. All coauthors discussed the results, L.V., J.-P. A. and S.P. wrote the manuscript with the input of all the authors.

\section{Supporting Information}

The Supporting Information is available free of charge on the ACS Publications website at

Evidence of absence of Spin Orbit Torques; discussion on field and current driven domain wall dynamics; influence of the non-adiabatic torque and of the damping parameter on the current-induced DW motion; damping and non-adiabatic torque in the particular case for which $\alpha=\beta$; simulations details.

\footnotetext{
${ }^{1}$ Manipatruni, S.; Nikonov, D. E.; Lin, C. C.; Gosavi, T. A.; Liu, H.; Prasad, B.; Huang, Y.-L.; Bonturim, E.; Ramesh, R.; Young, I. A. Scalable energy-efficient magnetoelectric spin-orbit logic. Nature 2019, 565 (7737), 35.

${ }^{2}$ Torrejon, J.; Riou, M.; Araujo, F. A.; Tsunegi, S.; Khalsa, G.; Querlioz, D.; Bortolotti, P.; Cros, V.; Yakushiji, K.; Fukushima, A.; Kubota, H.; Yuasa, S.; Stiles, M. D.; Grollier J. Neuromorphic computing with nanoscale spintronic oscillators. Nature 2017, 547 (7664), 428.

${ }^{3}$ Chakhmouradian, A. R.; Smith, M. P; Kynicky, J. From "strategic" tungsten to "green" neodymium: A century of critical metals at a glance. Ore Geology Reviews 2015, 64, 455-458 .

${ }^{4}$ Communication from the commission to the European Parliament, the Council, the European Economic and Social Committee and the Committee of the Regions on the 2017 list of Critical Raw Materials for the EU, Brussels, 13.9.2017 COM (2017) 490 Brussels

${ }^{5}$ Coey, J.; Smith, P. Magnetic nitrides. Journal of magnetism and magnetic materials 1999, 200, 405-424.

${ }^{6}$ Yasutomi, Y.; Ito, K.; Sanai, T.; Toko, K.; Suemasu, T. Perpendicular magnetic anisotropy of $\mathrm{Mn}_{4} \mathrm{~N}$ films on MgO (001) and $\mathrm{SrTiO}_{3}$ (001) substrates. Journal of Applied Physics 2014, 115, 17 A935.

${ }^{7}$ Meng, M.; Wu, S. X.; Ren, L. Z.; Zhou, W. Q.; Wang, Y. J.; Wang, G. L.; Li, S. W. Extrinsic anomalous hall effect in epitaxial $\mathrm{Mn}_{4} \mathrm{~N}$ films. Applied Physics Letters 2015, 106, 032407.

${ }^{8}$ Shen, X.; Chikamatsu, A.; Shigematsu, K.; Hirose, Y.; Fukumura, T.; Hasegawa, T. Metallic transport and large anomalous hall effect at room temperature in ferrimagnetic $\mathrm{Mn}_{4} \mathrm{~N}$ epitaxial thin film. Applied Physics Letters 2014, 105, 072410.

${ }^{9}$ Kabara, K.; Tsunoda, M. Perpendicular magnetic anisotropy of $\mathrm{Mn}_{4} \mathrm{~N}$ films fabricated by reactive sputtering method. Journal of Applied Physics 2015, 117, 17B512.

${ }^{10}$ W. J. Takei; R. R. Heikes; G. Shirane, Phys. Rev. 1962, 125, 1893.

${ }^{11}$ Emori, S.; Beach, G. S. D. Enhanced current-induced domain wall motion by tuning perpendicular magnetic anisotropy. Applied Physics Letters 2011, 98, 132508.

${ }^{12}$ Gushi, T.; Vila, L.; Fruchart, O.; Marty, A.; Pizzini, S.; Vogel, J.; Takata, F.; Anzai, A.; Toko, K.; Suemasu, T.; Attané, J. P. Millimeter-sized magnetic domains in perpendicularly magnetized ferromagnetic $\mathrm{Mn}_{4} \mathrm{~N}$ thin films grown on $\mathrm{SrTiO}_{3}$. Japanese Journal of Applied Physics 2018, 57, 120310.

${ }^{13}$ Moritz, J.; Rodmacq, B.; Auffret, S.; Dieny, B. Extraordinary hall effect in thin magnetic films and its potential for sensors, memories and magnetic logic applications. Journal of Physics D: Applied Physics 2008, 41, 135001.
} 
${ }^{14}$ Nguyen, V.D.; Torres, W.S.; Laczkowski, P.; Marty, A.; Jamet, M.; Beigné, C.; Notin, L.; Vila, L.; Attané, J.P. Elementary depinning processes of magnetic domain walls under fields and currents. Scientific reports 2014, 4, 6509.

${ }^{15}$ Boulle, O.; Malinowski, G.; Kläui, M. Current-induced domain wall motion in nanoscale ferromagnetic elements. Materials Science and Engineering: R: Reports 2011, 72, 159-187.

${ }^{16}$ Thiaville, A.; Rohart, S.; Jué, É.; Cros, V.; Fert, A. Dynamics of Dzyaloshinskii domain walls in ultrathin magnetic films. Europhysics Letters 2012, 100, 57002.

${ }^{17}$ Emori, S.; Bauer, U.; Ahn, S.-M.; Martinez, E.; Beach, G. S. Current-driven dynamics of chiral ferromagnetic domain walls. Nature materials 2013, 12, 611.

${ }^{18}$ Ryu, K.-S.; Thomas, L.; Yang, S.-H.; Parkin, S. Chiral spin torque at magnetic domain walls. Nature nanotechnology 2013, 8, 527.

${ }^{19}$ Hayashi, M.; Thomas, L.; Rettner, C.; Moriya, R.; Bazaliy, Y. B.; Parkin, S. S. Current driven domain wall velocities exceeding the spin angular momentum transfer rate in permalloy nanowires. Physical review letters 2007, 98, 037204.

${ }^{20}$ Uhlír, V.; Pizzini, S.; Rougemaille, N.; Novotný, J.; Cros, V.; Jiménez, E.; Faini, G.; Heyne, L.; Sirotti, F.; Tieg, C.; Bendounan, A.; Maccherozzi, F.; Belkhou, R.; Grollier, J.; Anane, A.; Vogel, J. Current-induced motion and pinning of domain walls in spin-valve nanowires studied by xmcd-peem. Physical Review B 2010, 81, 224418. ${ }^{21}$ Burrowes, C.; Mihai, A. P.; Ravelosona, D.; Kim, J. V.; Chappert, C.; Vila, L.; Marty, A.; Samson, Y.; GarciaSanchez, F.; Buda-Prejbeanu, L. D.; Tudosa, I.; Fullerton, E. E. ; Attané, J.-P. Non-adiabatic spin-torques in narrow magnetic domain walls. Nature Physics 2010, 6, 17.

${ }^{22}$ Cormier, M.; Mougin, A.; Ferré, J.; Thiaville, A.; Charpentier, N.; Piéchon, F.; Weil, R.; Baltz, V.; Rodmacq, B. Effect of electrical current pulses on domain walls in Pt/Co/Pt nanotracks with out-of-plane anisotropy: Spin transfer torque versus joule heating. Physical Review B 2010, 81, 024407.

${ }^{23}$ Curiale, J.; Lemaître, A.; Ulysse; C., Faini; G.; Jeudy, V. Spin drift velocity, polarization and current-driven domain-wall motion in (Ga, Mn)(As, P). Physical review letters 2012, 108, 076604.

${ }^{24}$ Adam, J.P.; Vernier, N.; Ferré, J.; Thiaville, A.; Jeudy, V.; Lemaître, A.; Thevenard, L.; Faini, G. Nonadiabatic spin-transfer torque in (Ga, Mn) As with perpendicular anisotropy. Physical Review B 2009, 80 (19), 193204.

${ }^{25}$ Miron, I. M.; Moore, T.; Szambolics, H.; Buda-Prejbeanu, L. D.; Auffret, S.; Rodmacq, B.; Pizzini, S.; Vogel, J.; Bonfim, M.; Schuhl, A.; Gaudin, G. Fast current-induced domain-wall motion controlled by the Rashba effect. Nature materials 2011, 10, 419.

${ }^{26}$ Yang, S.-H.; Ryu, K.-S.; Parkin, S. Domain-wall velocities of up to $750 \mathrm{~m} \mathrm{~s}-1$ driven by exchange-coupling torque in synthetic antiferromagnets. Nature nanotechnology 2015 10, 221.

${ }^{27}$ Caretta, L.; Mann, M.; Büttner, F.; Ueda, K.; Pfau, B.; Günther, C. M.; Hessing, P.; Churikova, A.; Klose, C.; Schneider, M.; Engel, D.; Marcus, C.; Bono, D.; Bagschik, K.; Eisebitt, S.; Beach, \& G. S. D. Fast current-driven domain walls and small skyrmions in a compensated ferrimagnet. Nature nanotechnology 2018, 13, 1154.

${ }^{28}$ Fukami, S.; Suzuki, T.; Ohshima, N.; Nagahara, K.; Ishiwata, N. Micromagnetic analysis of current driven domain wall motion in nanostrips with perpendicular magnetic anisotropy. Journal of Applied Physics 2008, 103, $07 E 718$.

${ }^{29}$ Szambolics, H.; Toussaint, J.-C.; Marty, A.; Miron, I. M.; Buda-Prejbeanu, L. Domain wall motion in ferromagnetic systems with perpendicular magnetization. Journal of Magnetism and Magnetic Materials 2009, 321, 1912-1918.

${ }^{30}$ Kim, K. J.; Kim, S. K.; Hirata, Y.; Oh, S. H.; Tono, T.; Kim, D. H.; Okuno, T.; Ham, W.S.; Kim, S.; Go, G.; Tserkovnyak, Y.; Tsukamoto, A.; Moriyama, T.; Lee, K.-J.; Ono T. Fast domain wall motion in the vicinity of the angular momentum compensation temperature of ferrimagnets. Nature materials 2017, 16, 1187.

${ }^{31}$ Komori, T.; Gushi, T.; Anzai, A.; Vila, L.; Attané, J.P.; Pizzini, S.; Vogel, J.; Isogami, S.; Toko, K.; Suemasu, T. Magnetic and magneto-transport properties of $\mathrm{Mn} 4 \mathrm{~N}$ thin films by Ni substitution and their possibility of magnetic compensation. Journal of Applied Physics 2019, 125 (21), 213902.

${ }^{32}$ Thiaville, A.; Nakatani, Y.; Miltat, J.; Suzuki, Y. Micromagnetic understanding of current-driven domain wall motion in patterned nanowires. Europhysics Letters 2005, 69, 990.

${ }^{33}$ Zhang, S.; Li, Z. Roles of nonequilibrium conduction electrons on the magnetization dynamics of ferromagnets. Physical Review Letters 2004, 93, 127204.

${ }^{34}$ Miao, M. S.; Herwadkar, A.; Lambrecht, W. R. Electronic structure and magnetic properties of $\mathrm{Mn}_{3} \mathrm{GaN}$ precipitates in Ga 1-x Mn $\times$ N. Physical Review B 2005, 72(3), 033204.

${ }^{35}$ Weisheit, M.; Fähler, S.; Marty, A.; Souche, Y.; Poinsignon, C; Givord, D. Electric field-induced modification of magnetism in thin-film ferromagnets. Science 2007, 315, 349-351. 
${ }^{36}$ Grupp, D. E.; Goldman, A. M. Giant piezoelectric effect in strontium titanate at cryogenic temperatures. Science 1997, 276, 392-394.

${ }^{37}$ Sidoruk, J.; Leist, J.; Gibhardt, H.; Sobolev, O.; Ouladdiaf, B.; Mole, R.; Eckold, G. Kinetics of domain redistribution in $\mathrm{SrTiO}_{3}$ under pulsed electric fields. Ferroelectrics 2016, 505, 200-209.

${ }^{38}$ Fontcuberta, J.; Skumryev, V.; Laukhin, V.; Granados, X.; Salje, E. K. Polar domain walls trigger magnetoelectric coupling. Scientific reports 2015, 5, 13784.

${ }^{39}$ Nakazawa, S.; Obinata, A.; Chiba, D.; Ueno, K. Electric field control of magnetic anisotropy in a Co/Pt bilayer deposited on a high- $\mathrm{KSrTiO}_{3}$. Applied Physics Letters 2017, 110, 062406.

${ }^{40}$ Ito, K.; Yasutomi, Y.; Kabara, K.; Gushi, T.; Higashikozono, S.; Toko, K.; Tsunoda, M.; Suemasu, T. Perpendicular magnetic anisotropy in co $\mathrm{x}$ mn4- $\mathrm{x} n(\mathrm{x}=0$ and 0.2$)$ epitaxial films and possibility of tetragonal mn4n phase. AIP Advances 2016, 6, 056201.

${ }^{41}$ Ito, K.; Higashikozono, S.; Takata, F.; Gushi, T.; Toko, K.; Suemasu, T. Growth and magnetic properties of epitaxial $\mathrm{Fe}_{4} \mathrm{~N}$ films on insulators possessing lattice spacing close to $\mathrm{Si}(001)$ plane. Journal of Crystal Growth 2016, 455, 66-70. 